\title{
Coherent adiabatic transport of atoms in radio-frequency traps
}

\author{
T. Morgan, B. O'Sullivan, and Th. Busch \\ Department of Physics, University College Cork, Cork, Ireland
}

\begin{abstract}
Coherent transport by adiabatic passage has recently been suggested as a high-fidelity technique to engineer the centre-of-mass state of single atoms in inhomogenous environments. While the basic theory behind this process is well understood, several conceptual challenges for its experimental observation have still to be addressed. One of these is the difficulty that currently available optical or magnetic micro-trap systems have in adjusting the tunneling rate time-dependently while keeping resonance between the asymptotic trapping states at all times. Here we suggest that both requirements can be fulfilled to a very high degree in an experimentally realistic setup based on radio frequency traps on atom chips. We show that operations with close to $100 \%$ fidelity can be achieved and that these systems also allow significant improvements for performing adiabatic passage with interacting atomic clouds.
\end{abstract}

\section{INTRODUCTION}

Going beyond nano-technologies and engineering quantum systems on the basis of single particles has in recent years been one of the most exciting and active areas of physics 1. Due to the fragile nature of single particle quantum states, quantum engineering techniques need to be fault tolerant and lead to high fidelities on every application to avoid the large and costly overhead that comes with error correction schemes [2]. One class of techniques that can achieve this are so-called adiabatic techniques and their use in optical systems has been widely investigated in the past. In particular, Stimulated Raman Adiabatic Passage (STIRAP) is one adiabatic technique that allows to transfer the populations from one electronic state to another with $100 \%$ fidelity [3]. It relies on the existence of a so-called dark state in a three level system and requires a counter-intuitive pulse sequence to coherently couple the individual levels.

Recently, it has been shown that similar techniques can, in principle, be used to control the quantised centreof-mass state of single particles [4 6. This atom-optical analogue has been dubbed Coherent Transport by Adiabatic Passage (CTAP) and while the possibility of observing this process has received significant attention [7, 8], the conditions that have to be fulfilled for its observation are currently hard to achieve experimentally. In particular, all states involved are required to be in resonance during the whole process. However, since the strength of the tunnel-coupling is usually adjusted by changing the distance between the microtraps, which leads to significant overlap of the neighbouring trapping potentials, the eigenstates become time-dependent. Several solutions to the problem have been suggested, with all involving significant experimental resources or restrictions on the parameter space [4, 6, 7]. A similar process coupling classical light between optical waveguides has recently been experimentally demonstrated 9 11].

Here we propose a simple experimental setup that fulfills all necessary conditions to observe CTAP for cold atoms. Our proposal is based on radio frequency (rf) traps, which have recently become one of the most versa- tile tools for trapping cold atoms [12, 13. The advantage of rf-systems is that their physics is well known, they are relatively benign systems to work with experimentally and are widely available today. They not only allow to create standard trapping potentials [12], but can also be used to coherently manipulate matter waves [13, 14] or create complicated, non-standard trapping geometries [15]17.

We will also show that our setup offers the possibility for extending adiabatic techniques to clouds of interacting atoms. The presence of interaction between the atoms introduces non-linearities into the system 18 which have been shown to inhibit the effectiveness of CTAP in transporting atoms [7. Several strategies to adjust the process and to allow transport in the presence of these non-linear interactions have been suggested, for example a fixed detuning between the potential wells [19. Here we will show that dynamically controlling the detuning between the potentials provides a marked improvement in the state transfer efficiency over both regular and fixed detuning CTAP.

In the following we will first briefly review the idea of CTAP for ultracold atoms. In section [II we will outline the theoretical description of rf-trapping and describe the system needed for CTAP. In section IV we demonstrate atomic transport in this system and show that the process allows high fidelity atomic transport in contrast to the intuitive method, which fails. In section $\mathrm{V}$ we examine the transport of an interacting atomic cloud and how the presence of non-linear interaction can be compensated for by dynamic detuning. Finally we conclude.

\section{COHERENT TRANSPORT ADIABATIC PASSAGE}

To briefly review the process of adiabatic population transfer let us consider a system of three ground states in three identical microtraps, $|0\rangle_{L},|0\rangle_{M}$ and $|0\rangle_{R}$ (see Fig. 11. In a linear arrangement the only tunnel couplings that are significant are $J_{L M}$ for the transition $|0\rangle_{L} \rightarrow$ $|0\rangle_{M}$ and $J_{M R}$ for $|0\rangle_{M} \rightarrow|0\rangle_{R}$. Assuming that the three states are in resonance when isolated, the Hamiltonian 


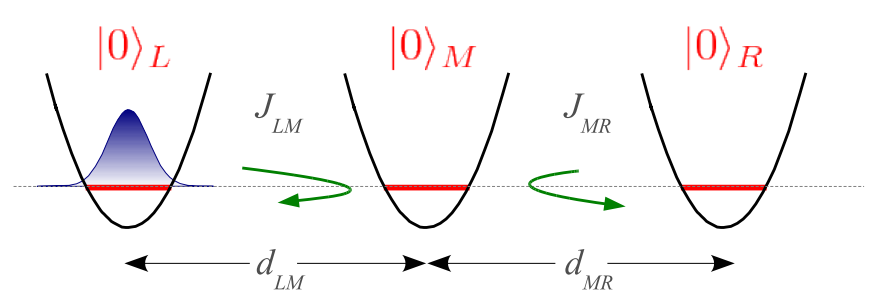

FIG. 1. Schematic of the CTAP process for an atom in the left trap. Reducing the distances between the traps leads to an increase in the tunnelling strengths.

for such a system is given by

$$
H(t)=\hbar\left(\begin{array}{ccc}
0 & -J_{L M}(t) & 0 \\
-J_{L M}(t) & 0 & -J_{M R}(t) \\
0 & -J_{M R}(t) & 0
\end{array}\right) .
$$

and a smooth time dependence of the tunnelling coupling pulses can be achieved by continuously changing the distances between the traps, $d_{L M}(t)$ and $d_{M R}(t)$. The eigenstates of this Hamiltonian are very well known [3] and of particular interest to our work here is the so-called dark state

$$
|d\rangle=\cos \theta|0\rangle_{L}-\sin \theta|0\rangle_{R}
$$

for which the mixing angle $\theta$ is given by

$$
\tan \theta=J_{L M} / J_{M R} .
$$

This state has a non-degenerate zero eigenvalue and therefore an adiabatic evolution will guarantee that the system, once prepared in $|d\rangle$, will always stay in it. Note that as the only contribution to $|d\rangle$ from the state $|0\rangle_{M}$ comes through the mixing angle, the system has zero probability to be found in $|0\rangle_{M}$ at any time.

The CTAP process can now be understood by considering an atom initially in the state $|0\rangle_{L}$. Increasing and decreasing $J_{M R}$ before $J_{L M}$, which is counter-intuitive to traditional tunneling schemes, continuously decreases the population in state $|0\rangle_{L}$ and increase the population in state $|0\rangle_{R}$, leading to a $100 \%$ transfer at the end of the process.

It is worth to stress again the conditions that have to be fulfilled for the above dynamics to occur. Firstly, the process must be adiabatic with respect to the energy level splitting in the harmonic oscillators, which means that the movement of the traps has to be slow and the whole process must take longer than $\omega_{\mathrm{HO}}^{-1}$, where $\omega_{\mathrm{HO}}$ is the harmonic oscillator frequency of the individual traps. As typical numbers of $\omega_{\mathrm{HO}}$ for microtraps are in the $\mathrm{kHz}$ regime, this means that the time required for this process is much shorter than lifetimes of the trapped atoms, which makes this process a promising tool for quantum information. The other condition we require, as previously mentioned, is that all single trap states are in resonance at any point in time, which is difficult to achieve once the trapping potentials start to overlap.
In the next section we will demonstrate how the second condition can be fulfilled in an experimentally realistic system using radio frequency potentials.

\section{RADIOFREQUENCY TRAPPING}

Radio frequency trapping relies on the process of coupling magnetic sublevels in the presence of an inhomogeneous magnetic field 12 15]. Consider a hyperfine atomic groundstate with total spin $F=\frac{1}{2}$. In the presence of the magnetic field the two hyperfine sublevels $m_{F}=\frac{1}{2}$ and $m_{F}^{\prime}=-\frac{1}{2}$ are split by an amount $\mu_{B} g_{F} m_{F} B$, where $g_{F}$ is the atomic g-factor of the hyperfine level and $\mu_{B}$ is the Bohr magneton. Irradiating such a system with a linearly polarized radio frequency, $\mathbf{B}_{\mathrm{rf}} \cos (\omega t)$, couples the sublevels $\left|\frac{1}{2}, \frac{1}{2}\right\rangle \leftrightarrow\left|\frac{1}{2},-\frac{1}{2}\right\rangle$ with spatial resolution due to the spatial dependence of the magnetic field. Here we will concentrate on a one dimensional description of such a process, which is valid when the radio frequency and magnetic field to be orthogonal to each other. Assuming the inhomogeneous magnetic field to be oriented in the $x$-direction, $B=B(x)$, the Hamiltonian of the coupled system can be written as

$$
H(x)=\frac{1}{2}\left(\begin{array}{cc}
\mu_{B} g_{F} B(x)-\hbar \omega & \hbar \Omega \\
\hbar \Omega & -\mu_{B} g_{F} B(x)+\hbar \omega
\end{array}\right),
$$

where the strength of the coupling is given by the Rabi frequency 20 .

$$
\Omega=\frac{\mu_{B} g_{F}}{4 \hbar}\left|\mathbf{B}_{\mathrm{rf}} \times \hat{e}_{B}\right| \sqrt{F(F+1)-m_{F} m_{F}^{\prime}},
$$

and where $\hat{e}_{B}$ is the orientation of the local static magnetic field. The eigenvalues of this Hamiltonian are [15]

$$
\begin{aligned}
E_{ \pm}(x) & = \pm \frac{1}{2} \sqrt{\hbar^{2} \Omega^{2}+\left[\mu_{B} g_{F} B(x)-\hbar \omega\right]^{2}} \\
& \approx \pm \frac{1}{2}\left[\mu_{B} g_{F} B(x)-\hbar \omega\right] \pm \frac{\hbar^{2} \Omega^{2}}{4\left[\mu_{B} g_{F} B(x)-\hbar \omega\right]}
\end{aligned}
$$

where the second expression is valid far from the resonance, $\hbar \Omega \ll\left[\mu_{B} g_{f} B(x)-\hbar \omega\right]$. The second term in the expression can be viewed as a Stark shift on the energy levels.

To create a multi-well potential it is necessary to use several frequencies and the above analysis will become significantly more complicated. However, if we assume that the individual frequencies are spaced sufficiently far apart and have low Rabi frequencies with respect to the detuning, we can approximate the dynamics locally by considering only the nearest resonance frequency, $\omega(x)=$ $\omega_{n(x)}$ [15. Formally this means that $n$ is chosen such that $\left[\mu_{B} g_{F} B(x)-\hbar \omega_{n(x)}\right]$ is minimized at any position $x$. The effects of the combined Stark shifts, produced by the frequencies not closest to resonance, can then be 
summed up as [15]

$$
L_{n}(x)=\sum_{j \neq n} \frac{\hbar^{2} \Omega^{2}}{4\left[\mu_{B} g_{F} B(x)-\hbar \omega_{j(x)}\right]},
$$

so that the eigenvalues are given by

$$
E_{ \pm}(x)= \pm \frac{1}{2} \sqrt{\hbar^{2} \Omega^{2}+\left[\mu_{B} g_{F} B(x)-\hbar \omega+2 L_{n}(x)\right]^{2}} .
$$

From this, and considering the that the couplings are strong enough to yield Landau-Zener transition probability close to unity, the resulting adiabatic potential is given by

$V_{a d, \pm}(x)=(-1)^{n(x)}\left[E_{ \pm}(x) \mp \frac{\hbar \omega_{n(x)}}{2}\right] \mp \sum_{k=1}^{n(x)-1}(-1)^{k} \hbar \omega_{k}$.

To produce a radio frequency potential with three minima along the $x$-direction we will need six different radio frequencies. In the following we will assume that the 1D linear magnetic field is given by $B(x)=b x$ where $b$ is the magnetic field gradient. For convenience we choose five of the six radio frequencies to be equally spaced initially, $\omega_{n}=2 n \pi \times 10000 \mathrm{kHz}(n=2: 6)$, which produces three equidistant minima. The value of the first radio frequency $\omega_{1}$ does need to have the same distance as the other frequencies, as its value only controls the height of the first maximum (see Fig. 2) and can therefore be adjusted without changing the trap geometry in the area where tunneling takes place. For our potential we set $\omega_{1}=1000 \mathrm{kHz}$ and in Fig. 2 we indicate the local frequencies and show the resulting adiabatic potential in the positive $x$-direction.

\section{ADIABATIC PASSAGE}

In this section we will apply the CTAP procedure to a single atom trapped in a three well rf-potential. We will show that the strong decay of the influence of the radio frequencies away from their respective resonance points allows us to fulfill the resonance condition between the asymptotic eigenstates at all times during the process. While the Stark shift from neighbouring resonances cannot be neglected, it is small enough to not destroy the process.

Movement of the traps is achieved by changing the individual radio frequencies that are associated with each trap. Traditionally for CTAP the middle trap is chosen to be at rest and the two outer ones are moving in and out (see also Fig. 1). Here we will choose a slightly different, but of course completely analogous, route in that we keep the position of the left trap fixed. This allows us to keep the values of the minima equal which is essential to satisfy the condition of resonance between all traps.

In order to achieve CTAP when moving the traps in this non traditional manner the approach of the right

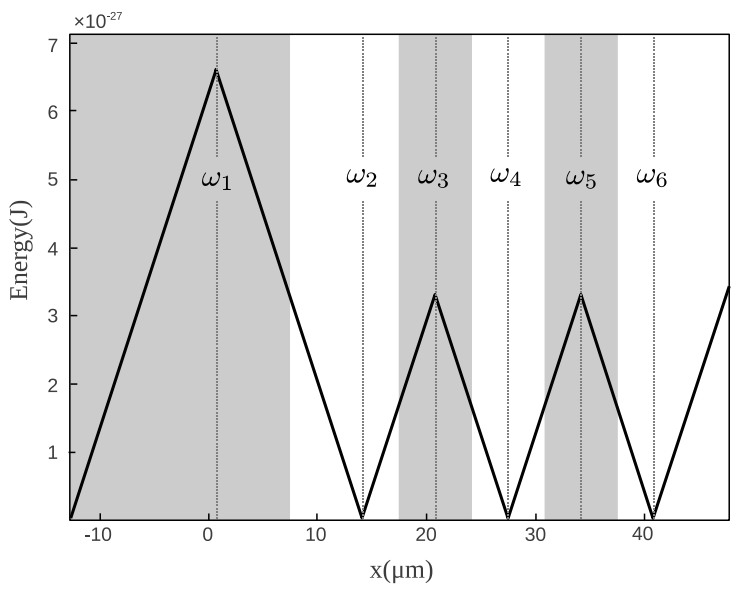

FIG. 2. Trapping potential created by six radio-frequencies $\omega_{1}=1000 \mathrm{kHz}$ and $\omega_{n}=2 \pi n \times 10000 \mathrm{kHz}, n=2: 6$. Their resonance-positions are marked by the broken vertical lines and the range over which they are applied is indicated by the grey and white zones. The magnetic field gradient has the strength $b=213 \mathrm{Gcm}^{-1}$ and $g_{F}=-\frac{1}{2}$ for the ${ }^{87} \mathrm{Rb}$ ground state ${ }^{2} S_{\frac{1}{2}}$. The Rabi frequency is chosen to be $2 \pi \times 50$ $\mathrm{kHz}$. The traps resemble harmonic oscillator potentials close to each minima.

trap towards the middle must start earlier than that of the approach of the middle trap to the left. One therefore initially only changes the frequencies $\omega_{5}$ and $\omega_{6}$, which determine the shape and position of the right hand side trap. After a delay $\tau$, the two frequencies $\omega_{3}$ and $\omega_{4}$ are changed as well, allowing to move the middle trap towards the left. Due to the adiabatic nature of the process the exact shape of this time-dependent frequency adjustment, $f(t)$, does not matter and we can formalise this process as

$$
\begin{aligned}
& \omega_{1}(t)=\omega_{1}\left(t_{0}\right), \\
& \omega_{2}(t)=\omega_{2}\left(t_{0}\right), \\
& \omega_{3}(t)=\omega_{3}\left(t_{0}\right)-\frac{1}{2} f(t-\tau) \theta(t-\tau), \\
& \omega_{4}(t)=\omega_{4}\left(t_{0}\right)-f(t-\tau) \theta(t-\tau), \\
& \omega_{5}(t)=\omega_{5}\left(t_{0}\right)-\frac{1}{2} f(t)-f(t-\tau) \theta(t-\tau), \\
& \omega_{6}(t)=\omega_{6}\left(t_{0}\right)-f(t)-f(t-\tau) \theta(t-\tau)
\end{aligned}
$$

where $\theta(t)$ is the Heaviside step function. In Fig. 3(a) these changes are shown for the typical system considered here and the resulting movements of the trap minima are displayed in Fig. 3(b). As can been seen, the minimum of the left trap remains stationary while the other traps are moving towards and away from it. The resulting movement between neighbouring traps exactly fulfills the requirement of the CTAP process, leading to the desired increase and decrease in the tunnelling strength between initially the middle and right traps before the increase 
(a)

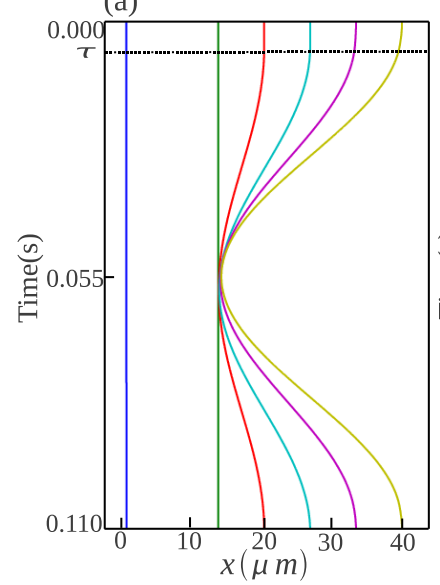

(b)

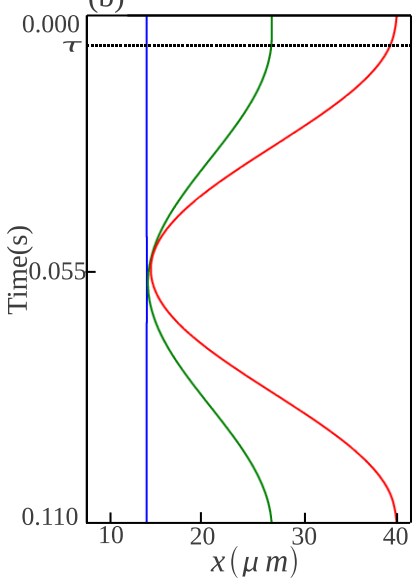

FIG. 3. (a) Radiofrequencies, $\omega_{n}$, as a function of time to achieve the counter-intuitive positioning sequence. (b) Positions of the trap minima as a function of time. The left trap remains stationary while the other two traps move towards it. The delay in the movement of middle trap in comparison to the right trap $(\tau=0.0055 \mathrm{~s})$ is indicated by the broken line.

and decrease in tunnelling strength between the left and middle traps.

To demonstrate adiabatic passage for single atoms and for typical experimentally realistic parameters, we will in the following show the results of numerical simulations of the full Schrödinger equation. We choose a single ${ }^{87} \mathrm{Rb}$ atom to be initially located in the centre-of-mass ground state of the left trap and start the process described in eq. (11) with an initial separation between the radio frequencies of $10000 \mathrm{kHz}$. The minimum distance to which the frequencies approach each other is $200 \mathrm{kHz}$, which ensures that we are always in the regime of tunnelling interaction, as the minimum barrier height between the individual traps is $5.3313 \times 10^{-29} \mathrm{~J}$ at the point of closest approach, compared to the ground state energy of $1.3615 \times 10^{-29} \mathrm{~J}$. The form of the adjustment function $f(t)$ is taken to be a cosine and for numerical simplicity we restrict ourselves to ones spatial dimension.

In Fig. 4 we show the probability density function with respect to time for the CTAP process. The overall time for this process is chosen to be $T=0.11 s$ which is large compared to the approximate harmonic oscillator frequency of the individual traps of $\omega_{\mathrm{HO}}^{-1} \approx 4 \times 10^{-6} \mathrm{~s}$, and we are therefore assured to be at all times in the dark eigenstate of the system. This can also be seen from the fact that the probability for being in the middle trap at any time is zero. The process leads to high fidelity population transfer and an absence of Rabi oscillations.

To compare the above situation to a process in which direct tunnelling between two neighbouring traps plays an important role, we show in Fig. 5 the results of the same process, this time however using an intuitive trapmovement. The direct tunnelling is clearly manifest in the appearance of Rabi oscillations between the traps and
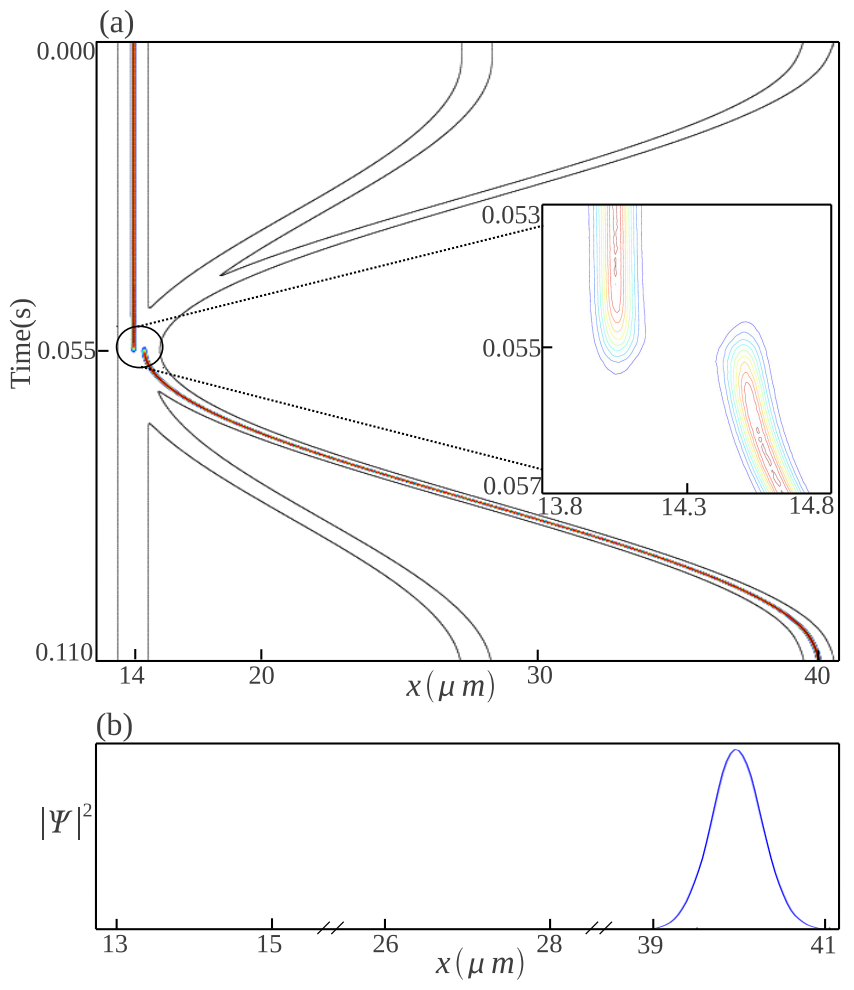

FIG. 4. (a) Probability density for a single atom initally located in the trap on the left hand side with respect to time for counter-intuitive trap movement. The inset shows the tunnelling area in greater detail. (b) Density of the final state in each of the three traps.

the process therefore does not deliver the required robust population transfer. In fact, the final state becomes highly susceptible to variations of the system parameters 21.

We have confirmed that these results are representative for a large range of parameters, making rf-traps ideal systems to investigate adiabatic processes in all generality.

\section{NON-LINEAR SYSTEMS}

The extension of adiabatic methods to non-linear systems is of large importance not only to describe experimental situations, but also for the understanding of the underlying physical principles [18, 19, 22, 23. In this section we show how CTAP can be used with timedependent potentials to coherently transport a cloud of interacting, Bose-condensed atoms. For this, we treat the adiabatic process as a series of stationary states which can be described by the time-independent GrossPitaevskii equation

$$
\mu \Psi(x)=\left(-\frac{\hbar^{2}}{2 m} \nabla^{2}+V(x)+g_{1 D}|\Psi|^{2}\right) \Psi(x),
$$




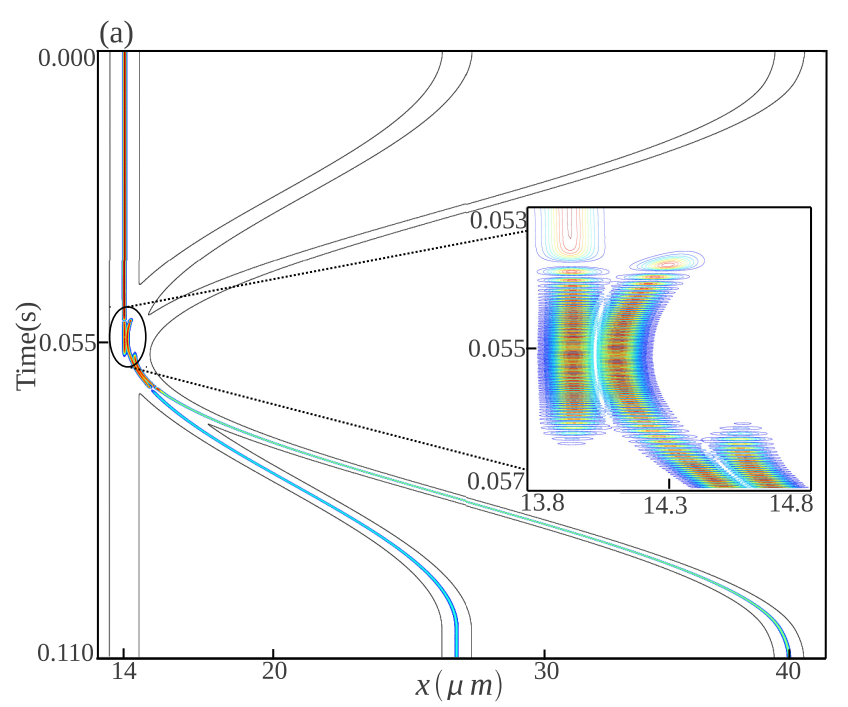

(b)

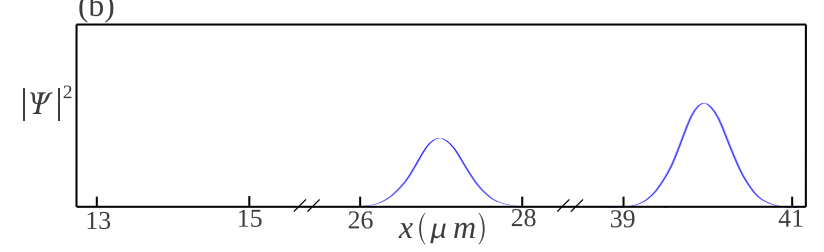

FIG. 5. (a) Probability density for a single atom initally located in the trap on the left hand side with respect to time for intuitive trap movement. The inset shows the tunnelling area in greater detail, where Rabi oscillations between neighbouring traps are clearly visible. (b) Density of the final state in each of the three traps.

where $V(x)$ is the external and $\mu$ the chemical potential at each respective point in time. The onedimensional interaction strength between bosons with a three-dimensional s-wave scattering length $a_{s}$ is given by and $g_{1 D}=\frac{4 N \hbar^{2} a_{s}}{m a_{\perp}}\left(a_{\perp}-C a_{s}\right)^{-1}$ [24]. The trap width in the radial direction is given by $a_{\perp}$ and $C \approx 1.4603$. In the three level approximation the Hamiltonian can therefore be written as

$$
H(t)=\hbar\left(\begin{array}{ccc}
\hbar \omega_{L}+\mu_{L} & -J_{L M}(t) & 0 \\
-J_{L M}(t) & \hbar \omega_{M} & -J_{M R}(t) \\
0 & -J_{M R}(t) & \hbar \omega_{R}+\mu_{R}
\end{array}\right),
$$

where $\mu_{L, R}$ are the chemical potentials associated with the atomic clouds in left or right trap and $\omega_{L, M, R}$ are the harmonic oscillator frequencies associated with the individual traps. Because the particle number in each individual trap is a function of time, the associated change in the chemical potentials will detune the resonance between the energies. To be able to compensate for this we will in the following allow for the trapping frequencies to be functions of time as well. Starting with a cloud of atoms in the left trap and then attempting to perform CTAP, it is clear that the chemical potential $\mu_{L}$ will decrease over time, while $\mu_{R}$ will increase. As at all times the uncoupled traps have to be in resonance, one can see

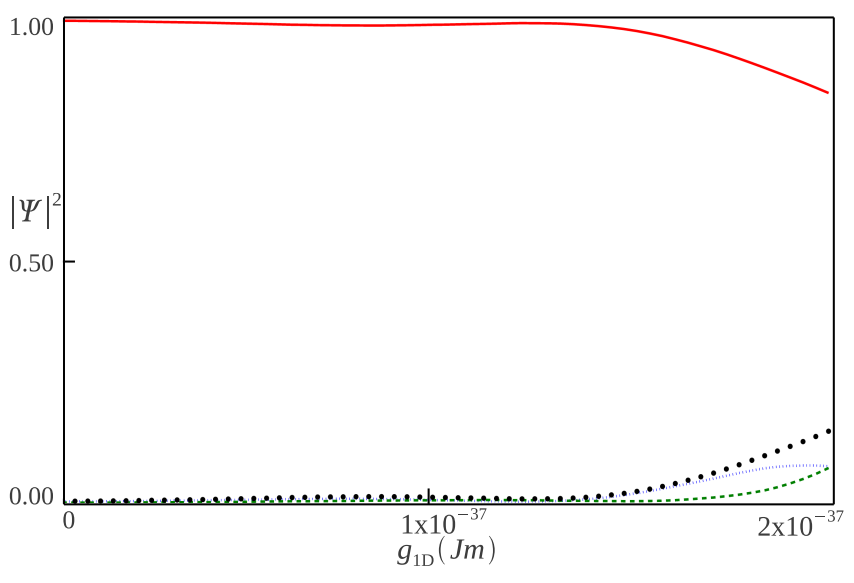

FIG. 6. Final population in left (verticle dashed line, blue), middle (horizontal dashed line, green) and right (solid line, red) traps with increasing interaction strength. The dotted black line shows the total population not occupying the target (right) trap. The maximum value of $g_{1 D}$ corresponds to $\mu=$ $1.4318 \times 10^{-29} \mathrm{~J}$ which is smaller than $\hbar \omega_{L, M, R}$ at all times.

that a time-dependent adjustment of the trapping frequencies $\omega_{L, R}$ can allow us to compensate for this change. However, in order to be able to make the three state approximation, we need to make sure that $\mu<\hbar \omega_{L, M, R}$ at all times, for all values of $\mu$ and $\omega_{L, M, R}$. This means in practice that the process is limited to cold atomic clouds with small non-linearities.

Using the same radio frequency potential as in the linear case, we place a cloud of interacting ${ }^{87} \mathrm{Rb}$ atoms in the ground state of the left trap by determining the solution to the Gross-Pitaevskii equation for an isolated trapping potential. To show the influence of the non-linear behaviour, we first carry out the same counter-intuitive trap movements as in the linear section without timedependent change in the trapping frequencies. In Fig. 6 we show the final populations in the individual traps as a function of increasing values for $g_{1 D}$. It is immediately obvious that even for weak interactions the non-linear term is disruptive to the process of CTAP. In fact, for $g_{1 D}=2 \times 10^{-37} \mathrm{Jm}$ the state transfer efficiency is reduced to $84 \%$. Choosing a typical radial trap width of $130 \mathrm{~nm}$, this value of $g_{1 D}$ corresponds to $N=2$ for ${ }^{87} \mathrm{Rb}$.

As we have already stated above, a possible strategy to combat this effect of changing system energies in the individual traps is to adjust the individual trapping frequencies. To restore resonance for a changing chemical potential one can adjust the trapping frequency to make sure that at any point in time $\hbar \omega_{L}(t)+\mu_{L}(t)=\hbar \omega_{M}=$ $\hbar \omega_{R}(t)+\mu_{R}(t)$. However, determining the required adjustments is not a simple exercise for at least two reasons. First, the density dependence of the chemical potential will prevent this change from simply being linear in time and, secondly, a conceptual difficulty in determining the individual chemical potentials arises when the traps are 
close together. While one could try to calculate the chemical potential, and therefore the on-site energies, in all traps at all times to a good approximation, this is certaintly not experimentally possible. In the following, we therefore suggest a simple functional form for dynamically detuning the outer traps and we show that it allows us to achieve significantly higher transfer than possible without adjustments. A similar idea, however without time-dependence, was recently proposed by Graefe et al. [19], who showed that by detuning the left and the right trap by the same fixed amount throughout the process an improved transfer of population can be achieved.

The outline of our scheme for dynamic detuning is as follows. Initially the cloud is trapped in the left trap which we detune such that resonance with the eigenstates of the other two traps is ensured (since the traps are far apart, it is possible to determine the chemical potential $\left.\mu_{L}\right)$. As we time evolve the system tunnelling sets in and we begin to reduce the detuning on the left trap to zero while increasing the detuning of the right trap, as atoms enter it. This can be achieved by adjusting the radio frequencies $\omega_{2}$ and $\omega_{6}$, associated with the left and right hand side trap, respectively. Here we suggest that a good form of function for the adjustment related to the left hand side trap is

$$
\Delta \omega_{2}(\kappa ; \tilde{t})=\frac{1}{2}[1-\tanh (\kappa \tilde{t})] \Delta \omega_{0}
$$

where the inital value for the change in $\omega_{2}$ is given by $\omega_{0}$. The function runs between $\Delta \omega_{0}$ and 0 and the steepness in the crossover region is determined by $\kappa$. This gives us an effective handle on both, the time when the adjustment starts, and the duration of the adjustment (see inset of Fig. 7). Here $\tilde{t}=t-T / 2$, with $T$ being the overall duration of the process. At the same time the frequency of the right hand side trap needs to be adjusted as well and it is easy to see that a mirror symmetric change in $\omega_{6}$ is the best choice.

$$
\Delta \omega_{6}(\kappa ; \tilde{t})=\frac{1}{2}[1+\tanh (\kappa \tilde{t})] \Delta \omega_{0}
$$

The dynamic adjustments of the radio frequency equations (11) then become

$$
\begin{aligned}
\omega_{1}(t) & =\omega_{1}\left(t_{0}\right) \\
\omega_{2}(t) & =\omega_{2}\left(t_{0}\right)-\Delta \omega_{2}(\kappa, \tilde{t}) \\
\omega_{3}(t) & =\omega_{3}\left(t_{0}\right)-\frac{1}{2} f(t-\tau) \theta(t-\tau) \\
\omega_{4}(t) & =\omega_{4}\left(t_{0}\right)-f(t-\tau) \theta(t-\tau) \\
\omega_{5}(t) & =\omega_{5}\left(t_{0}\right)-\frac{1}{2} f(t)-f(t-\tau) \theta(t-\tau) \\
\omega_{6}(t) & =\omega_{6}\left(t_{0}\right)-f(t)-f(t-\tau) \theta(t-\tau)+\Delta \omega_{6}(\kappa, \tilde{t})
\end{aligned}
$$

In Fig. 7 we show the final population transferred to the right trap for increasing values of $\kappa$ and for $\Delta \omega_{0}=1.5$

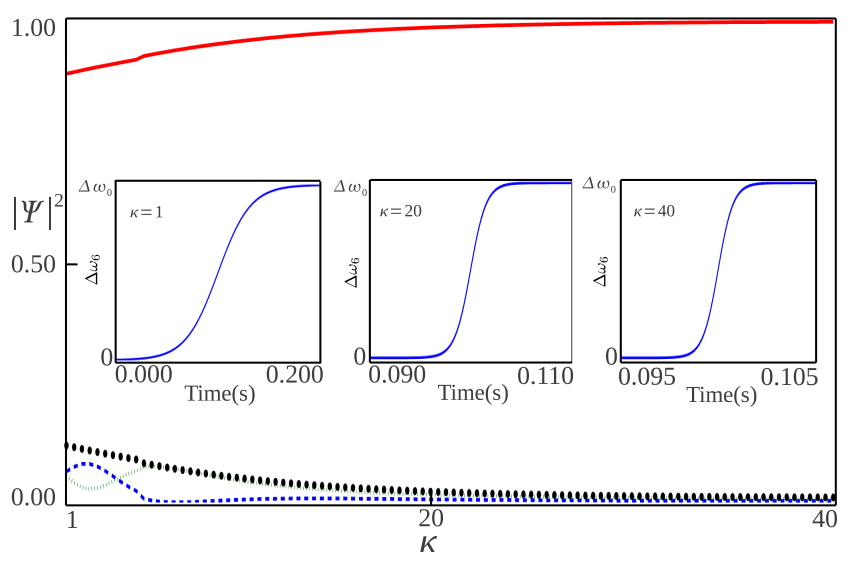

FIG. 7. Final population in left (verticle dashed green line), middle (horizontal dashed blue line) and right (solid red line) traps for non-linear CTAP with increasing $\kappa$ and $\Delta \omega_{0}=1.5$ $\mathrm{kHz}$. The dotted black line shows the total population not occupying the target (right) trap. The insets show the shape of $\Delta \omega_{6}(\kappa ; \tilde{t})$ for different values of $\kappa$. An increased value of $\kappa$ increases the time when the adjustment begins and decreases the adjustment time.

$\mathrm{kHz}$. We can see that the dynamic adjustment of the detunings of the outer traps allows us to achieve population transfer of $>99 \%$, up from $84 \%$. This is an improvement over both standard CTAP and fixed detuning in the weak interaction regime and, in fact, returns to the transfer efficiency of single particle CTAP.

\section{CONCLUSIONS}

We have shown that radio frequency traps can be used as microtraps for processes in which an adjustable tunneling strength is required. Neighbouring trapping potentials can be overlapped without significantly changing the underlying energy level structure. This property has allowed us to create a triple well radio frequency potential in which coherent transport using adiabatic passage can be demonstrated. For a single atom, it was shown that complete transfer between the left and right traps by utilizing the dark state of the system is possible, maintaining the advantages of an absence of Rabi oscillations and robustness against variation in system parameters.

For a cloud of weakly interacting atoms we have demonstrated a technique that significantly improves the efficiency of CTAP by dynamically detuning the outer traps. Our suggested setup is close to experimental realities, avoids the large overhead of other suggestions and can easily be extended to other adiabatic techniques. 


\section{ACKNOWLEDGEMENTS}

The authors would like to thank Peter Krüger and Thomas Fernholz for valuable discussions. This work was supported by Science Foundation Ireland under project numbers 05/IN/I852 and 10/IN.1/I2979. BOS. acknowledges support from IRCSET through the Embark Initiative No. RS/2006/172.
[1] M.A. Nielsen, and I.L. Chuang, Quantum Computation and Quantum Information, Cambridge University Press, Cambridge (2000).

[2] A.M. Steane, Phys. Rev. Lett. 77, 793 (1996).

[3] K. Bergmann, H. Theuer, and B.W. Shore, Rev. Mod. Phys. 70, 1003 (1998).

[4] K. Eckert, M. Lewenstein, R. Corbalán, G. Birkl, W. Ertmer, and J. Mompart, Phys. Rev. A 70, 023606 (2004).

[5] A.D. Greentree, J.H. Cole, A.R. Hamilton, and L.C.L. Hollenberg, Phys. Rev. B 70, 235317 (2004).

[6] K. Eckert, J. Mompart, R. Corbalán, M. Lewenstein, and G. Birkl, Opt. Comm. 264, 264 (2006).

[7] M. Rab, J.H. Cole, N.G. Parker, A.D. Greentree, L.C.L. Hollenberg, and A.M. Martin Phys. Rev. A 77, 061602 (2008).

[8] B. O'Sullivan, P. Morrissey, T. Morgan, and Th. Busch, Phys. Scr. T140, 014029 (2010).

[9] S. Longhi, Phys. Rev. E 73, 026607 (2006).

[10] S. Longhi, G. Della Valle, M. Ornigotti, and P. Laporta, Phys. Rev. B 76 201101R (2007).

[11] A.A. Rangelov, N.V. Vitanov, and B.W. Shore, J. Phys. B 42, 055504 (2009).

[12] O. Zobay, B.M. Garraway, Phys. Rev. Lett. 86, 1195 (2001).
[13] T. Schumm, S. Hofferberth, L.M. Andersson, S. Wildermuth, S. Groth, I. Bar-Joseph, J. Schmiedmayer and P. Krüger, Nature Physics 1, 57 (2005).

[14] S. Hofferberth, B. Fischer, T. Schumm, J. Schmiedmayer, and I. Lesanovsky, Phys. Rev. A 76, 013401 (2007).

[15] Ph.W. Courteille, B. Deh, J. Fortágh, A. Günther, S. Kraft, C. Marzok, S. Slama, C. Zimmermann, J. Phys. B: At. Mol. Opt. Phys. 39, 1055 (2006).

[16] I. Lesanovsky, T. Schumm, S. Hofferberth, L. M. Andersson, P. Krüger, and J. Schmiedmayer. Phys. Rev. A. 73, 033619 (2006).

[17] T. Fernholz, R. Gerritsma, P. Krüger, R.J.C. Spreeuw, Phys. Rev. A 75, 063406 (2007).

[18] J. Liu, B. Wu, Q. Niu, Phys. Rev. Lett. 90, 170404 (2003)

[19] E. M. Graefe, H. J. Korsch, D. Witthaut, Phys. Rev. A. 73, 013617 (2006).

[20] W. Ketterle, and N.J. van Druten, N. J.Adv. Mol. Opt. Phys. 37, 181 (1996).

[21] J. Mompart, K. Eckert, W. Ertmer, G. Birkl, and M. Lewenstein Phys. Rev. Lett. 90, 147901 (2003).

[22] A. Smerzi, S. Fantoni, S. Giovanazzi, and S. R. Shenoy, Phys. Rev. Lett. 79, 4950 (1997).

[23] I. Marino, S. Raghavan, S. Fantoni, S.R. Shenoy, and A. Smerzi, Phys. Rev. A. 60, 487 (1999).

[24] M. Olshanii, Phys. Rev. Lett. 81, 938 (1998). 